\title{
Catheter ablations of supraventricular tachycardias
}

\author{
Misikova S, Stancak B, Sudzinova A \\ Eastern Slovakia Institute of Cardiac and Vascular Diseases, Kosice, Slovakia. smisikova@vusch.sk
}

\begin{abstract}
Background: Supraventricular tachycardias (SVT) occur at all ages. Their spectrum as well as tolerance change with increasing age.

Objectives: The aim of our study was to assess the course of SVT catheter ablations in elderly patients when compared to younger population.

Methods: The course of the SVT radiofrequency catheter ablation (RFCA) in the group of 288 patients, who underwent catheter ablation at our department during 24 months was retrospectively evaluated. Patients were divided into two subgroups according to age. In the first group 142 patients younger than 65 years were included and 146 patients aged 65 years or more in the second group. In both groups, the occurrence of heart rhythm disorders, fluoroscopy time and the incidence of complications with varying severity were observed. Results: Life-threatening complications were observed only in three patients from the first group (one pulmonary embolism and two unintended complete atrioventricular blocks). Serious complications included a haemopericardium requiring a pericardial drainage in two cases and an arterial-venous fistula in one patient in the first group, and one retroperitoneal heamatoma in the second group. Minor complications represented by one heamatoma larger than $5 \times 5 \mathrm{~cm}$ occurred in one patient from each group. The occurrence of complications as well as average fluoroscopy time were not correlated between the groups.

Conclusion: Based on our experience, radiofrequency catheter ablation of SVT in elderly patients is a safe procedure when indicated correctly. Taking into account the physical status of patients in this age group it is advisable to simplify and thus shorten the procedure (Tab. 1, Ref. 13). Full Text in PDF www.elis.sk.

Key words: radiofrequency ablation, supraventricular tachycardia, elderly patiens.
\end{abstract}

Supraventricular tachycardias (SVT) occur among all age groups. Their spectrum as well as their tolerance changes with increasing age. Many forms, which are well tolerated in adults, are accompanied by dizziness, presyncope or syncope, chest pain or dyspnea in elderly. However, poor response to SVT attacks in older subjects is due to cardiopulmonary and other comorbidities when compared to younger population (1). The reason why pharmacological therapy is used more frequently than the catheter ablation in the management of SVT, is the polymorbidity present in most elderly subjects. Because of the ever-increasing life expectancy, the optimal approach to treatment ofSVT will eventually change in this age group.

The aim of our study was to assess the course of catheter ablation of SVT in elderly patiens when compared to younger population, and whether higher age of patiens may increase procedural complications.

\section{Patients and methods}

The course of the SVT radiofrequency catheter ablation (RFCA) in the group of 288 patients, who underwent catheter ablation at our department during 24 months were evaluated. Pa-

Eastern Slovakia Institute of Cardiac and Vascular Diseases, Kosice, Slovakia

Address for correspondence: S. Misikova, MD, Bukova 5, SK-040 14, Kosice, Slovakia.

Phone: +421.55 .7891312 , Fax: +421.55 .7891313$ tients were divided into two groups according to age. In the first group 142 patients younger than 65 years were included (mean age $49.5 \pm 7.2$ years, 61 men and 81 women), and 146 patients aged 65 years or more in the second group (mean age $73.9 \pm 8.9$ years, 69 men and 77 women). In both groups, the occurrence of heart rhythm disorders, fluoroscopy time and the incidence of complications with varying severity were observed.

The electrophysiology study was performed in the standard electrophysiology laboratory. A decapolar catheter was advanced through the right subclavian vein towards the coronary sinus, and via the right femoral vein other catheters were introduced. A multipolar halo catheter was used in patients with atrial flutter for mapping the reentry circle around the tricuspid annulus. In patients with other types of SVT, catheters were further advanced into the right atrium, right ventricle and to the registration of the His bundle potential. 4-20 polar catheters with a fixed or steerable tip inserted under fluoroscopic control were used. Intracardiac recorder (Prucka General Electric) was used for registration intracardiac activity, using ECG filtration of $30-500 \mathrm{~Hz}$, and paper recording speed of $100-200 \mathrm{~mm} / \mathrm{s}$. Electric stimulation was performed using external stimulator Biotronik UHS 20. Stimuli of $1 \mathrm{~ms}$ width at twice the measured threshold were applied. Radiofrequency energy was applied through ablation catheters (IBI or Biosense-Webster) with 4-8 mm long tip. The power source was the RF generator Medtronic Atakr II. Nonfluoroscopic mapping system Localisa was used during ablation. 
Complications that may accompany catheter ablation procedures, were divided according to their severity into three categories.

1. Major or life-threatening complications including: myocardial infarction, embolic stroke, bradycardia - induced polymorphic ventricular tachycardia, peripheral embolism, valve impairment or unintended complete atrioventricular block.

2. Serious complications including: deep vein thrombosis, internal bleeding and drainage-requiring pericardial effusion.

3. Minor complications including: haematoma $(>5 \times 5 \mathrm{~cm})$, decrease of the haemoglobine level by more than $1 \mathrm{~g} / \mathrm{l}$, transient alteration in mental state after sedation, or angina pectoris without myocardial infarction requiring interruption of the procedure (2).

\section{Statistical analysis}

Data are expressed as mean \pm standard deviations. The differences among groups were analyzed by ANOVA. A probability value of $\mathrm{p}<0.05$ was accepted as statistically significant.

\section{Results}

Atrioventricular nodal reentry tachycardia (AVNRT) or atrioventricular reentry tachycardia (AVRT) were more often observed in younger patients, in contrast, atrial fibrillation was dominant among elderly patients. The difference in the incidence of atrial tachycardia or flutter was not significant between the two groups of patients.

Overview of the post procedural complications is present in Table 1. Life-threatening complications were observed only in patients from the first group. One patient developed signs of pulmonary embolism and was successfully treated by systemic thrombolysis. Unintended complete atrioventricular (AV) block in two patients with AVNRT following ablation of the slow pathway was observed. In one patient, a second ablation was performed due to the presence of slow pathway conduction in difficult anatomic conditions. In the other patient, the block was relatively well tol-

Tab. 1. Occurrence of complications in both groups according to severity.

\begin{tabular}{lccc}
\hline Complication & $<65$ years & $\geq 65$ years & difference \\
\hline Life threatening & - & - & NS \\
Death & - & - & \\
Myocardial infarction & - & - & \\
Bradycardia induced VT & 1 & - & \\
Pulmonary embolism & - & - & \\
Embolic stroke & 2 & - & NS \\
Unintended AVB III. grade & - & - & \\
Valve impairment & & & \\
\hline Serious & - & - & \\
Deep vein thrombosis & - & 1 & \\
Retroperitoneal bleeding & 2 & - & \\
Pericardial effusion & 1 & - & \\
Arterial - venous fistula & & & \\
\hline Minor & - & - & \\
Haematoma $(>5 \times 5$ cm) & - & - & \\
Sedation leading to alteration & - & & \\
Angina pectoris & &
\end{tabular}

erated with stable escape rhythm, though several months later, a permanent pacemaker was implanted because of physical activity intolerance. Serious complications included haemopericardium requiring pericardial drainage in two cases and arterial-venous fistula in one patient who was surgically treated (in the group of patients $<65$ years old), and a retroperitoneal haematoma due to vascular wall impairment in one patient in the older group. Minor complications represented by one heamatoma larger than $5 \times 5 \mathrm{~cm}$ occurred in one patient from each group. The occurrence of complications as well as average fluoroscopy time (692 \pm 539 and 549 \pm 436 seconds) were not correlated $(p=N S)$.

\section{Discussion}

The physiology of cardiovascular system in older population differs in some aspects from younger patients. The ageing process is associated with a decrease in the total number of pacemaker cells in the conduction system, also calcifications or amyloid penetration (3). These pathophysiologic mechanisms subsequently modify the electrophysiologic characteristics of the atrial conduction system. Accessory pathways also change as age increases, the result is prolongation of their effective refractory period (4). This potentially leads to slower ventricular rate during SVT and atrial fibrillation paroxysms. A small incidence of sudden cardiac death in elderly patients with accessory pathway also confirms the above mentioned fact (5). Destruction of cardiomyocytes by apoptosis and the higher incidence of ischaemic heart disease and arterial hypertension in these patients is related to augmentation of the fiber interstitium. It results in lower compliance and increased myocardial stiffness, leading to dispersion of the atrial refractoriness (6) that explains the higher incidence of atrial fibrillation in elderly patients. Impairment of diastolic relaxation and left ventricle filling are the reasons why even lower ventricular frequencies during the SVT paroxysm are worse tolerated in elderly patients. This leads to reduction of cardiac output causing dyspnea or chest pain also in patients without history of cardiovascular disease $(7,8)$. A higher incidence of preexisting cerebrovascular diseases and worsening of cardiovascular reflexes are the reasons, why high ventricular frequencies during the SVT paroxysm may lead to critical reduction in the cerebral blood flow and the corresponding symptomatology (9).

The physiologic process of ageing is also associated with higher appearance of other diseases, which is related more frequently to polypragmasy. Interaction of drugs with antiarrhythmics increases the risk of pro-arrhythmic complications. Several factors influence the higher susceptibility of the geriatric heart towards the antiarrhythmic effects. Conditions that commonly accompany elderly people are worsened status of liver and kidneys, decreased blood proteins, higher incidence of dehydration, hypokalemia and hypomagnesaemia (10). Pharmacologic treatment of SVT paroxysm is accompanied by negative inotropic and hypotensive effects of antiarrhythmics.

Only a few articles were found in the literature dealing with this problem $(11,12)$. No differences were described in the occurrence of the complications during SVT ablations between 
older and younger population. Our results were similar to these studies.Smith (13) in 2001 published the total number of RFCA procedures in the USA between the years 1991-1998. With increasing life expectancy, the number of ablations in elderly people rises. Elderly patients form and for the future will still form an important part of patients undergoing the catheter ablation. Frequent hospital admissions, and the prolonged usage of antiarrhythmics favour catheter ablation compared to pharmacology treatment.

Although radiofrequency ablation plays a profitable role in SVT management, it carries a number of risks that could possibly lead to serious complications. In elderly patients, it is important to consider the higher risk of right ventricular or atrial perforation precipitated by thinning of the myocardial wall. Degenerative diseases of the connective tissue and bones may worsen the tolerance of the procedure, in extreme cases resulting in earlier termination. Generalized arteriosclerosis may also complicate the venous and arterial approach during catheterization. Dehydration, to which elderly patients are already susceptible, increases the risk of thrombembolic events. However, peri-procedural care reduces the risk of these complications and ultimately improves the outcome.

\section{Conclusion}

Based on our experience, radiofrequency catheter ablation of SVT in elderly patients is a safe procedure when indicated correctly. Even though older patients usually suffer from other comorbidities increasing the risk of any invasive procedure, the results of our study showed similar risk in both groups of patients. Taking into account the physical status of patient, it is advisable to simplify and thus shorten the procedure. Application of analgetics or anxiolytics and even adjustment of the examination bed may improve patient's tolerability. Furthermore, an adequate psychological preparation of the patient and a constant verbal contact during the procedure are equally important. These measures lead to a better prognosis and lower number of post-procedural problems.

\section{References}

1. Epstein LM, Chiesa N, Wong MN et al. Radiofrequency catheter ablation in the treatment of supraventricular tachycardia in the elderly. J Am Coll Cardiol 1994; 23: 1356-1362.

2. Zado ES, Callans DJ, Gottlieb CD et al. Efficacy and safety of catheter ablation in octogenarians. J Am Coll Cardiol 2000; 35: 458-462.

3. Ascherman M, Korbel F, Bytešník J. Kardiovaskulární systém v stáŕí. Kardiologie. Galen. 1343-1355.

4. Klein GJ, Yee R, Sharma AD. Longitudinal electrophysiologic assessment of asymptomatic patiens with the Wolff-Parkinson-White electrophysiologic pattern. N Engl J Med 1989; 320: 1229-1233.

5. Wellens HJ, Durrer D. Wolff-Parkinson-White syndrome and atrial fibrillation. Relation between refractory period of accessory pathway and ventricular rate during atrial fibrillation. Am J Cardiol 1974; 34: 777-782.

6. Stančák B. Patofyziologické mechanizmy predsieňových porúch rytmu I. Predsieňový flutter. Vnitř Lék 1998; 44(12): 722-726.

7. Gerstenblith G, Fleg JL, Becker LC et al. Maximum left ventricular filling rate in healthy individuals measured by gated blood pool scans: effect of age. Circulation 1984; 53, 586-595

8. Burch GE, DePasquale NP. Geriatric cardiology. Am Heart J 1969; 78: 700-715.

9. Nixon JV, Hallmark H, Page K et al. Ventricular performance in human hearts aged 61 to 73 years. Am J Cardiol 1985; 56: 932-941.

10. Verbeeck RK, Cardinal JA, Wallace SM. Effect of age and sex on the plasma binding of acidic and basic drugs. Eur J Clin Pharmacol 1984; 27: 91-98.

11. DaCosta A, Zarqane-Sliman N, Romeyer-Bouchard C et al. Safety and efficacy of radiofrequency ablation of commom atrial flutter in elderly patiens: a single center prospective study. PACE 2003; 26, 1729-1734.

12. Kihel J, DaCosta A, Kihel A et al. Long-term efficacy and safety of radiofrequency ablation in elderly patiens with atrioventricular nodal reentrant tachycardia. Europace 2006; 8: 416-420.

13. Smith PN, Vidaillet H, Sharma PP et al. Catheter ablation in the elderly in the United States: use in the medicare population from 1991 to 1998. PACE 2001; 24: 66-69. 